Delft University of Technology

\title{
A rugged FBG-based pressure sensor for water level monitoring in dikes
}

Schenato, Luca; Aguilar-Lopez, Juan Pablo; Galtarossa, Andrea; Pasuto, Alessandro; Bogaard, Thom; Palmieri, Luca

DOI

10.1109/JSEN.2021.3067516

Publication date

2021

Document Version

Final published version

Published in

IEEE Sensors Journal

\section{Citation (APA)}

Schenato, L., Aguilar-Lopez, J. P., Galtarossa, A., Pasuto, A., Bogaard, T., \& Palmieri, L. (2021). A rugged FBG-based pressure sensor for water level monitoring in dikes. IEEE Sensors Journal, 21(12), 1326313271. [9381888]. https://doi.org/10.1109/JSEN.2021.3067516

\section{Important note}

To cite this publication, please use the final published version (if applicable).

Please check the document version above. 
Green Open Access added to TU Delft Institutional Repository

'You share, we take care!' - Taverne project

https://www.openaccess.nl/en/you-share-we-take-care

Otherwise as indicated in the copyright section: the publisher is the copyright holder of this work and the author uses the Dutch legislation to make this work public. 


\title{
A Rugged FBG-Based Pressure Sensor for Water Level Monitoring in Dikes
}

\author{
Luca Schenato $^{\circledR}$, Member, IEEE, Juan Pablo Aguilar-López, Andrea Galtarossa ${ }^{\circledR}$, Fellow, IEEE, \\ Alessandro Pasuto, Thom Bogaard, and Luca Palmieri ${ }^{\circledR}$, Member, IEEE
}

\begin{abstract}
This paper describes the implementation of an FBG sensor to measure water levels in a dike. The sensor is based on a 3D-printed mechanical transducer through which the external pressure is converted into longitudinal strain exerted on the fiber. An additional FBG integrated within the sensor measures temperature and is used to compensate for the temperature effects on the first FBG. By employing an aluminum alloy case, the sensor is suitable for operations in harsh environments and rough installation procedures. Four sensors of this kind have been successfully tested on a real scale dike at the Water Proof Holland facility in The Netherlands.
\end{abstract}

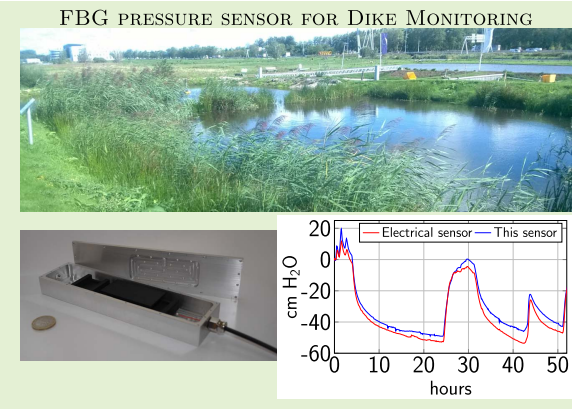

Index Terms-Fiber gratings, optical fiber sensors, pressure sensors, sensor systems and applications, soil.

\section{INTRODUCTION}

$\mathbf{T}$ HE measurement of pressure is essential in many different application fields, from automotive [1] to structural health monitoring in civil structures [2] as well as biomechanical applications [3], [4]. It also plays a paramount role in environmental monitoring, particularly for riverbanks and dikes stability [5]. In that regard, the pressure directly influences the stresses exerted by the soil, which may trigger the slope stability failures. Also, seepage forces occur due to any variation of the hydrostatic pressure within the dike foundation, leading to the progressive removal of fine soil particles. Ultimately, this is the basis of the backward erosion piping mechanism, which may cause the structure to collapse [6]. High spatial and pressure resolution over a small pressure range is recommended in this application [7], [8].

Manuscript received March 3, 2021; accepted March 12, 2021. Date of publication March 19, 2021; date of current version June 14, 2021. This work was supported in part by the Project DOMINO through the European Commission (Horizon 2020), in part by the Italian Ministry of Instruction, University and Research, and in part by The Netherlands Organisation for Scientific Research (NWO) within the Water JPI and the WaterWork2014 Cofunded Call. The associate editor coordinating the review of this article and approving it for publication was Dr. Anuj K. Sharma. (Corresponding author: Luca Schenato.)

Luca Schenato and Alessandro Pasuto are with the Research Institute for Geo-Hydrological Protection, National Research Council, 35137 Padua, Italy (e-mail: luca.schenato@cnr.it).

Juan Pablo Aguilar-López and Thom Bogaard are with the Faculty of Civil Engineering and Geosciences, Delft University of Technology, 2628 CD Delft, The Netherlands (e-mail: j.p.aguilarlopez@ tudelft.nl).

Andrea Galtarossa and Luca Palmieri are with the Department of Information Engineering, University of Padua, 35121 Padua, Italy (e-mail: luca.palmieri@unipd.it).

Digital Object Identifier 10.1109/JSEN.2021.3067516
In the last 30 years, many environmental sensing problems have been addressed using optical fiber sensors [9]-[11], including pressure measurement. Single-point [12]-[14] as well as distributed [15]-[17] fiber optic sensors have been proposed for pressure measurement, but the most explored are undeniably, the fiber Bragg grating (FBG) based sensors [14]. Indeed, fiber optic sensors, including FBGs, can offer attractive features such as intrinsic safety, immunity to electromagnetic fields, remote sensing and powering, multiplexing capabilities, and ruggedness [18].

Traditional pressure sensors, mostly based on standpipe piezometers, electrical strain gauges, and vibration wires, yet effective, struggle to work in harsh environments. Long-term reliability and stability, often in the presence of electromagnetic interference (EMI), are rather difficult to achieve with standard sensors. What makes FBGs outperforming traditional sensors is the ability to enable long-distance multi-point pressure measurements. This feature is impossible to achieve with traditional sensors, neither over a short nor a long distance.

One of the main challenges that has been tackled during the development of these sensors is the intrinsic small pressure sensitivity of bare FBGs, estimated at $3.04 \mathrm{pm} / \mathrm{MPa}$, which is too low for any practical pressure application [19]. Therefore, some authors proposed to enhance the pressure sensitivity by embedding the FBG in a thick polymer layer and exploiting the Poisson's effect [20]-[24]. Others envisaged attaching the FBG to a flexible structure, like a diaphragm, which deforms when exposed to pressure, dragging the FBG [25]-[28]. The use of FBGs inscribed in polymer optical fibers (POF), with lower Young's modulus than silica glass, has been proven 
to guarantee large sensitivity, despite considerable hysteresis [29]. Recently, an additional sensitivity boost with a large tunability range and limited hysteresis, has been achieved by the adoption of special POFs, with even lower stiffness than standard POFs [30], [31].

Another issue common to all FBG-based pressure sensors is the temperature cross-sensitivity, mostly addressed by encasing an additional FBG into the sensor in a strain-free configuration [32], [33]. In that way, this second FBG is sensitive only to the temperature. The strain-free condition is not mandatory for temperature compensation, as it is sufficient for the two FBGs having different pressure and temperature sensitivities. For example, Leal-Junior et al. [34] proposed a compensation technique for the temperature cross-sensitivity on an oblong diaphragm-based FBG sensor. The method was based on extensive analytical modeling of the sensor behavior for the temperature and pressure variations. In such a way, the cross-sensitivity between the pressure and temperature can be greatly reduced. The same research group also proposed an analytical technique for estimating the temperature and pressure with a single FBG on the oblong diaphragm-based FBG sensor. The method was based on the analysis of both parameters' transient behavior, in the case of slowly-varying temperature [35]. Other solutions consist of using long FBGs, exposed to the pressure only over a short section while the rest can be used for temperature compensation [36], [37]. Most recently, a temperature-insensitive scheme employing two FBGs embedded at opposite sides of the same diaphragm has also been proposed [38].

The sensor here proposed implements an FBG cemented to a flexible mechanical transducer produced by additive manufacturing; it also embeds a second FBG for temperature compensation. Other FBG-based pressure sensors, implemented with 3D-printing technology, have been recently proposed. For example, Lin et al. [39] designed and characterized a $3 \mathrm{D}$-printed cylindrical pressure sensor, not temperature compensated, embedding an FBG in a partitioned case. The case's inner wall undergoes bending when exposed to pressure, inducing a strain to the FBG. This design, yet effective, exposes the FBG to the external environment, compromising the sensor's long-term operability. Hong et al. [40] proposed a vertical pressure sensor by merely embedding an FBG inside a polylactic acid (PLA) block during the printing process. No temperature compensation mechanism has been either implemented. Yet very cheap, quick and easy to be produced and customized, it was not suitable for a harsh environment.

Most importantly, it is essentially a force sensor, not suitable for hydrostatic pressure measurement. Similar limitations impair another 3D-printed force sensor proposed by the same author [41], where a cylindrical vessel encases a vertical plastic rod, embedding an FBG, that undergoes bulging when compressed. Moreover, all these sensors were not sensitive enough for the application addressed here, which requires resolution at the $\mathrm{cm}$-scale.

The sensor of this study is apt to work in harsh environments, covered with soil and submerged in water, and has a sensitivity of several $\mathrm{pm} / \mathrm{cm} \mathrm{H}_{2} \mathrm{O}$. Since the mechanical transducer is made by $3 \mathrm{D}$ printing, the cost of the proposed sensor, of a few hundred USD per single unit, is practically determined by the cost of the aluminum case and the two FBGs. Further, it offers some distinctive unattainable advantages, typical of FBG sensors. The device can be used for real-time monitoring, and can be remotely operated and powered; moreover, it can be daisy-chained, greatly simplifying its deployment. Given the device's quasi-distributed nature, the system integrator is not required to accurately define and choose the exact location of each sensing point. Of course, the interrogator impacts the overall system costs, but the system becomes exceptionally competitive in terms of cost per single sensing point when several sensors are deployed.

This paper extends previously reported preliminary results [42], and it is organized as follows. Section II describes the design of the sensor and the implementation of the prototype. The sensors' calibration is reported in Section III, where the sensitivity to pressure and cross-sensitivity to temperature is determined. The validation of the sensors on the real-field application is described in Section IV. Eventually, the conclusions are drawn in Section V.

\section{SEnsor Design}

The sensor proposed in this work, is a rugged version of the quasi-distributed pressure sensor (QDPS) introduced in Ref. [43], designed to address the challenges of an underground field installation, e.g., along a dike. That sensor was entirely produced by additive manufacturing (HP Multi jet-fusion technology with HP 3D High Reusability PA12 plastic). Consequently, that prototype was not sturdy enough to withstand underground installation in a dike. When buried in the soil, the QDPS would have sensed the underground pore water pressure and an equivalent pressure related to the above soil's weight. Moreover, being made entirely of plastic, the sensor was also somewhat flexible, causing variability in the pressure readout depending on the externally applied forces. For these reasons, we have optimized the design of the former sensor to make it sturdier.

A 3D exploded view with a cross-section of the rugged sensor, with all its components, is shown in Fig. 1a. Given the successful operation of the preliminary version, the main transducing mechanism has been only minimally changed to fit an external aluminum case. The pressure-transducing mechanism is realized with a hexagonal pantograph with the top pad designed to be orthogonally loaded by the external pressure. An optical fiber crosses the pantograph, and it is glued at the pantograph's wedges. The portion of the fiber between the cemented points, underneath the pad, has an FBG inscribed in it $\left(\mathrm{FBG}_{\mathrm{P}}\right)$. Therefore, when a load is applied to the pad, the pantograph exerts a strain on the fiber, read by that FBG. In the pantograph's inner space, under the pad and with the fiber running through them, two end-stop vertical pillars limit the pad's compression under high load and prevent the structure from damage in case of excessive pressure. The same fiber crosses an adjacent open structure, and it is glued at its walls. An additional grating, $\mathrm{FBG}_{\mathrm{T}}$, is written in the fiber portion between those two walls. This structure is designed to be mechanically uncoupled to the pressure-sensitive part, and, therefore, $\mathrm{FBG}_{\mathrm{T}}$ is used to measure only the temperature and 


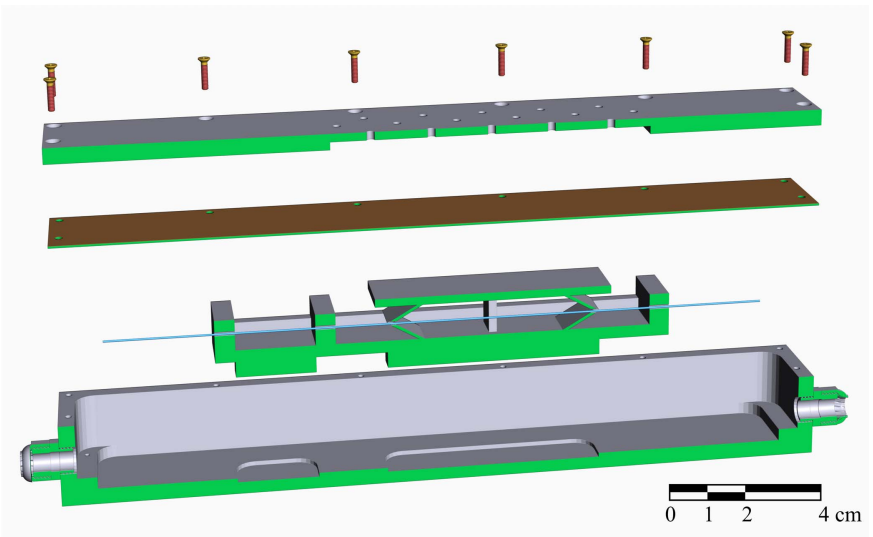

(a)

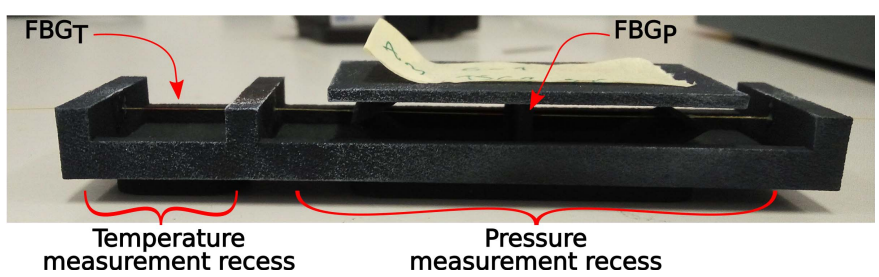

(b)

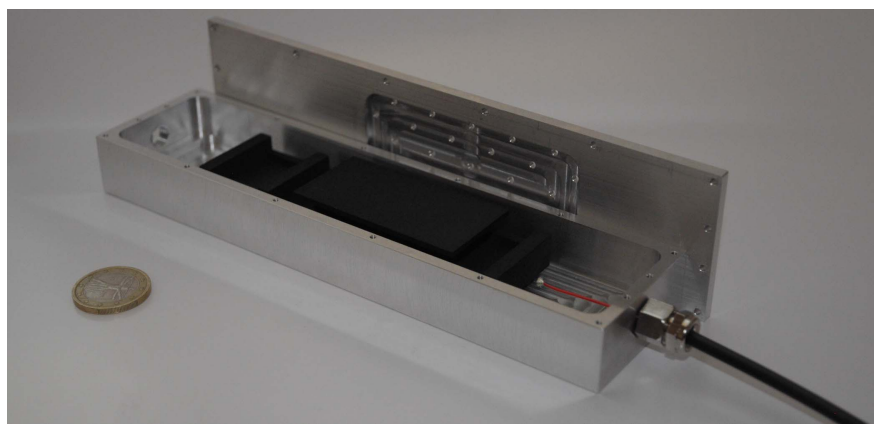

(c)

Fig. 1. (a) Cross-section of the exploded sensor CAD model (from the top to the bottom: the top cover, the sealing rubber sheet, the transducer and the aluminum case); (b) a picture of the 3D-printed transducer; (b) the rugged QDPS with the open case and top cover (c).

cross-compensate the thermal effect on the FBGP. In particular, the pantograph, the recess for temperature measurement, and the supporting structure have been $3 \mathrm{D}$ printed in a single component (see Fig. 1b).

The geometrical parameters of the transducer were numerically optimized in the previous version and remain mostly unchanged here: in particular, the pad measures $30 \times 60 \times 2 \mathrm{~mm}$, and the transducer's arms are $30^{\circ}$-angled and $0.7 \mathrm{~mm}$-thick; the inner pantograph height is $9 \mathrm{~mm}$. The recess with $\mathrm{FBG}_{\mathrm{T}}$ is $20 \mathrm{~mm}$ long, and the overall length of the $3 \mathrm{D}$ printed structure is $115 \mathrm{~mm}$. At the bottom of the transducer, two extruded pads have been added to assist the alignment of the transducer and to prevent any movement inside the case, as explained below. The 3D-printed transducer is therefore placed inside a watertight aluminum case, whose walls are approx. $4 \mathrm{~mm}$ thick to withstand the installation procedure and the operational conditions. The external dimensions of the case is $48 \times 25 \times 192 \mathrm{~mm}$; it is long enough to allow locating
TABLE I

Nominal Central Wavelength of the FBGs of Each QDPS

\begin{tabular}{|l|l|l|l|l|}
\hline QDPS ID & $\mathrm{E}_{1}$ & $\mathrm{~S}_{1}$ & $\mathrm{~S}_{2}$ & $\mathrm{~S}_{3}$ \\
\hline CWL FBG $_{\mathrm{T}}(\mathrm{nm})$ & 1528 & 1544 & 1576 & 1552 \\
CWL FBG $_{\mathrm{P}}(\mathrm{nm})$ & 1534 & 1550 & 1582 & 1556 \\
\hline
\end{tabular}

two empty slots, at the front and back, where the fiber excess coils can be accommodated. In such a way, the sensor can be adapted to have the fiber entering and exiting the case either from the same or opposite sides. To align and keep in place the transducer, two grooves, matching the bottom extruded pads of the transducer, have been milled at the case's internal bottom surface. To further immobilize the transducer, the extruded pads have been glued in the grooves. Two threaded holes are drilled through the short walls of the case, where IP69 cable glands are screwed for the watertight sensor cabling.

The top cover, screwed to the case using a set of regularly spaced screws, is grooved by face-milling above the pad, in the inner surface. The groove is then drilled by several holes. The lid and the case clamp a $0.5 \mathrm{~mm}$-thick rubber sheet, directly in contact with the pad, which seals the device at the case walls and functions as a pressure-sensitive flexible membrane. The holes in the lid groove, mentioned above, couple the rubber sheet and the underneath pad with the external pressure. Furthermore, when used in saturated soil, water should fill the gap in the lid groove. The bare optical fiber is finally inserted in an armoured cable sheath clamped via IP69 cable glands at the lateral holes. The open case with the transducer and the lid is shown in Fig. 1c. Please note that the rubber sheet is not shown in the picture.

In the present version of the QPDS device, the fiber enters and exits the case through the same side because the sensors have been installed vertically in a bore drilled on top of the dike to mimic the standard site surveys of standpipe piezometers. Four of these sensors have been employed in the field test. Given that the preliminary prototype showed that $\mathrm{FBG}_{\mathrm{P}}$ has a larger temperature sensitivity than $\mathrm{FBG}_{\mathrm{T}}$, in the rugged sensor, differently from the prototype of [43], the central wavelength (CWL) of the $\mathrm{FBG}_{\mathrm{P}}$ has been fixed some nm longer than that of the $\mathrm{FBG}_{\mathrm{T}}$. The nominal CWLs of the FGBs encased in the installed sensors have been reported in Tab. I.

\section{Calibration}

The calibration of the rugged QDPSs has been performed in a main chamber connected to a water reservoir and hosting up to 5 sensors at a time. The main chamber is not sealed, but it is equipped with a tube high enough to reach the required pressure and large enough to let optical and electrical connectors through (see Fig. 2). The main chamber and the water reservoir are flasks made with PVC pipes (diameter $20 \mathrm{~cm}$ ), sealed at the bottom with a drain test plug and connected with a hose. The top of the main flask is extended with a narrower PVC pipe $(\varnothing 5 \mathrm{~cm})$, long enough to achieve the required test pressure; the same pipe acts as an outlet for the sensors patch cords. The flasks have a capacity of about 10 liters. 


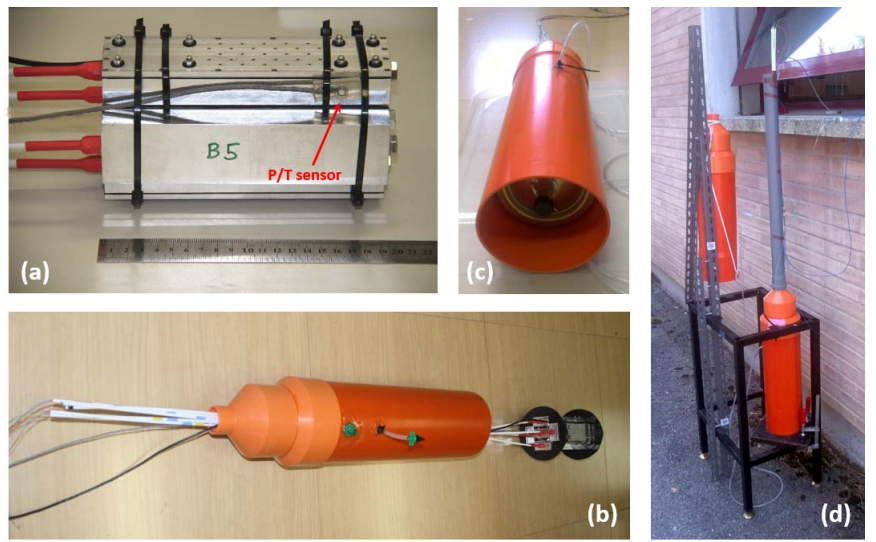

Fig. 2. (a) Rack of QDPSs assembled for characterization; arrow indicates the reference pressure and temperature electric sensor. (b) The rack of QDPSs ready for the installation inside the testbed main flask. (c) Detail of the steel drain test plug used to seal the bottom of the flasks. (d) Operational experimental setup with the extension tube installed on the main flask to achieve the required pressure; the hose connecting the flasks is barely visible.

The pressure at which the sensors were exposed was controlled by varying the head difference between the main chamber and the water reservoir. Differently, the temperature in the testbed is not actively controlled. It was varied by starting the calibration with cold water and letting it naturally warm up to ambient temperature. This temperature calibration procedure allowed us to cover the expected temperature fluctuation for the field test. Nonetheless, given the materials properties and construction details, the sensors are expected to work well beyond this range (e.g., from a few to $40-50{ }^{\circ} \mathrm{C}$ ). The pressure range expected to be required for the field test was about $80 \mathrm{~cm}$ of $\mathrm{H}_{2} \mathrm{O}$; therefore, the first three sensors $\left(\mathrm{S}_{1}, \mathrm{~S}_{2}\right.$, and $\mathrm{S}_{3}$ ) were calibrated all together up to about a standard water head of $1 \mathrm{~m}$. A second calibration cycle involved the sensor $\mathrm{E}_{1}$, and was performed up to an extended water head of about $1.4 \mathrm{~m}$ to investigate the design robustness against overpressure. In particular, the sensor $E_{1}$ was implemented by applying a large pre-strain to the FBGp to contrast the expected significant deformation of the transducer at higher pressure.

The central wavelength shifts of the FBGs were measured with a commercial interrogator (Micronoptics ${ }^{\circledR}$ Hyperion si155), while the actual pressure and temperature were monitored by a reference pressure and temperature $(\mathrm{P} / \mathrm{T})$ electric probe (MS5803-02BA from TE ConnectivityTM).

Fig. 3 shows the domain of the stimuli (pressure and temperature) applied to the sensors during the calibration, while Fig. 4 shows the sensors response. In all plots, one may notice that the amplitude of wavelength variation of the pressure FBG is almost independent of temperature, whereas the average of these variations tends to increase with temperature. This is consistent with the fact that as the temperature increases, the structure expands and the CWL of FBGp red-shifts; at the same time, however, the temperature has a smaller impact on the mechanical response of the sensor to pressure.

Each sensor's response was modeled by a two 2-variate function, providing the CWL shift of each FBG with respect to the pressure and temperature fields with the functions
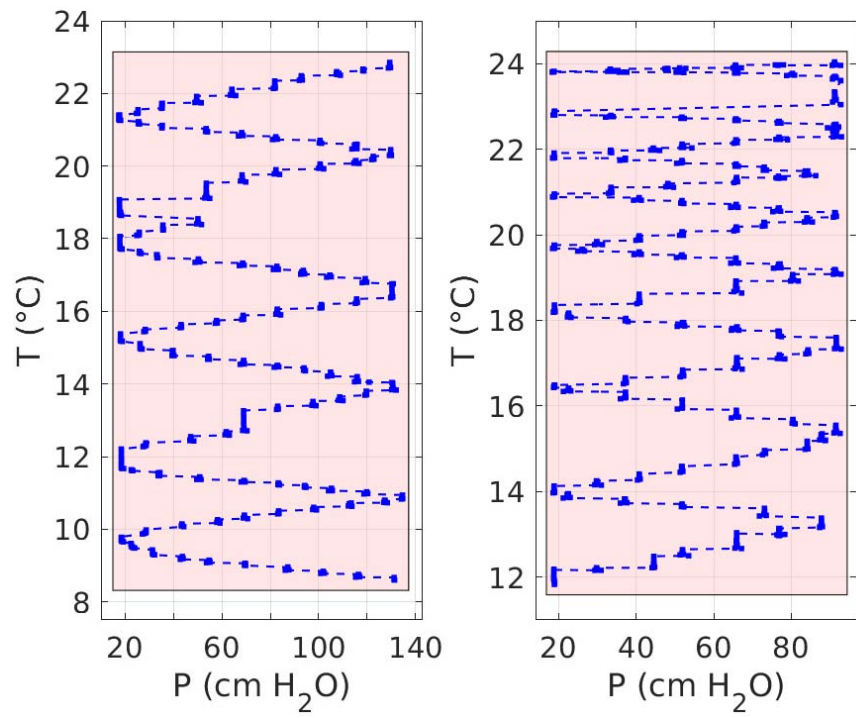

Fig. 3. Calibration domain of the QDPS $E_{1}$ (left plot), and $S_{1}, S_{2}$, and $S_{3}$ (right plot). Blue dots represent the actual calibration points, the dashed lines indicate the temporal evolution of the measurements (starting from lower temperatures), and the pink area represents the considered calibration domain.
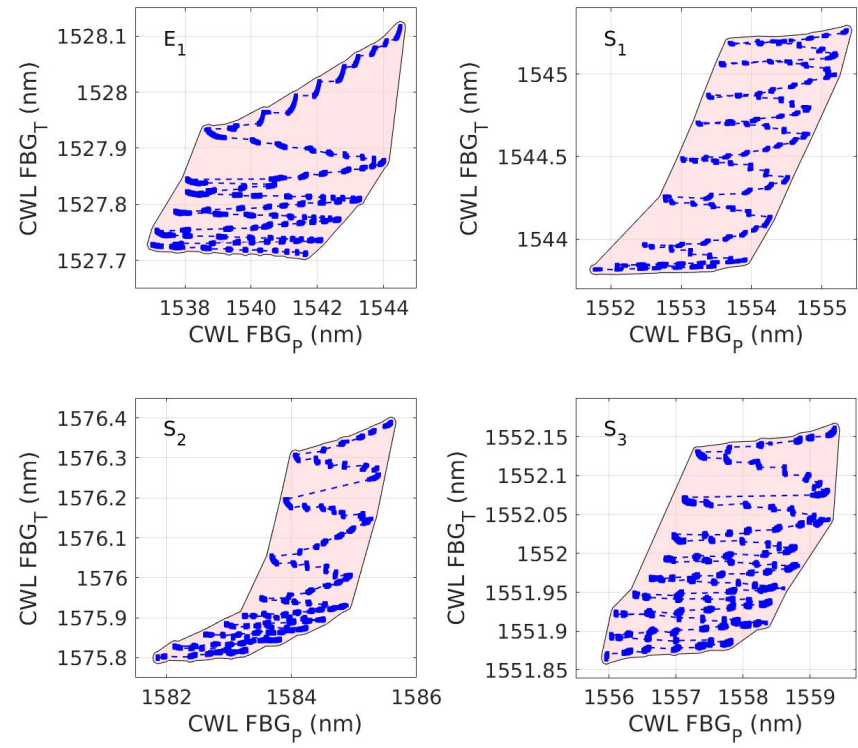

Fig. 4. The responses of the QDPSs to the calibration stimuli. Blue dots represent the actual response to the calibration points, the dashed lines indicate the temporal evolution of the measurements, and the pink area represents the considered response domain.

$f_{P}(P, T)$ of the pressure FBGs set to second-order polynomials.

Fig. 5a shows the corresponding interpolation surfaces of $f_{P}(P, T)$ for the sensors, obtained by least squares estimation (LSE), where dots represent the measured data. As one may notice, the response of FBGp was slightly nonlinear, especially for pressure, and with a more marked non-linearity for the sensor $\mathrm{E}_{1}$.

The modeling of the temperature FBGs required more care. After completing the calibration cycles, we noted that $\mathrm{FBG}_{\mathrm{T}}$ responses were largely independent of pressure (as expected by design) but have two distinctively different behaviors with respect to temperature (see Fig. 5b). This feature raises as 

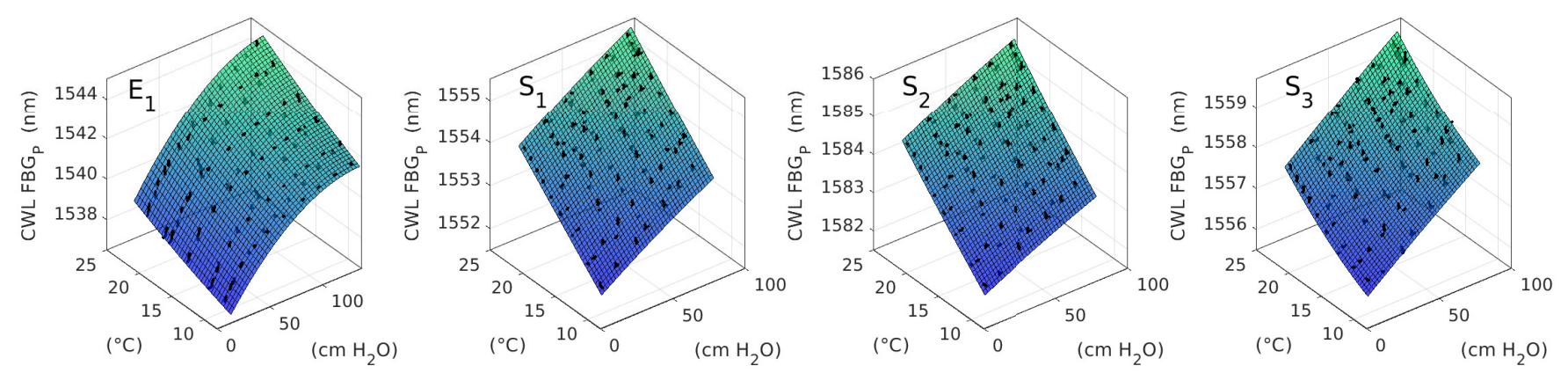

(a)
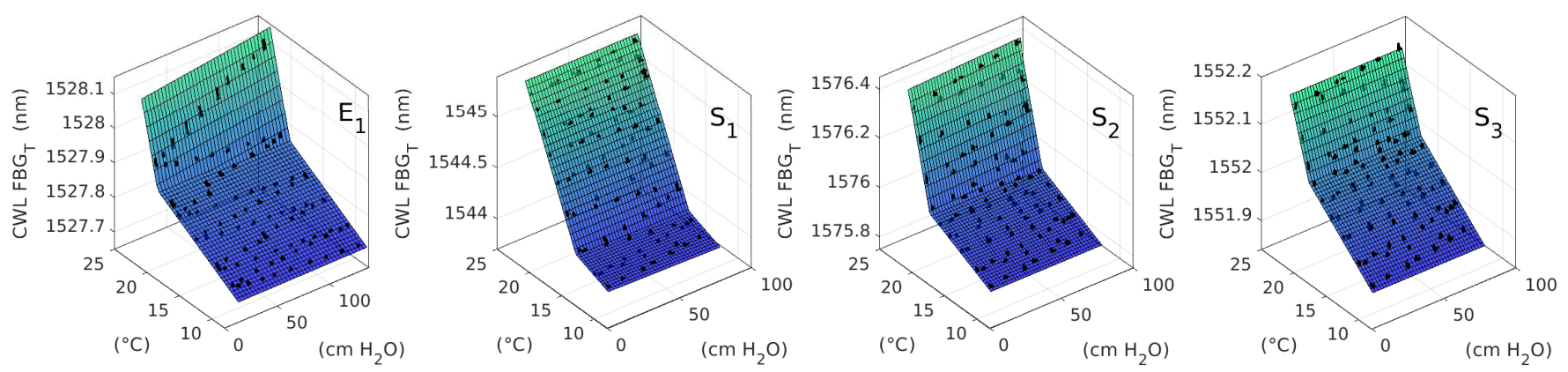

(b)

Fig. 5. The response surfaces of $\mathrm{FBG}_{\mathrm{P}}(\mathrm{a})$ and $\mathrm{FBG}_{\mathrm{T}}(\mathrm{b})$ of the QDPSs. Dots are the experimental points; surfaces are the 2nd order polynomial interpolants.

we anchored the temperature FBGs at the supporting walls with no pre-strain during the implementation to avoid that the thermal expansion of the structure might stretch the temperature FBGs; however, the fiber's slack was not enough. Actually, at lower temperature, the temperature FBGs were not strained, and hence, their response was determined by the thermo-optic effect of the fiber. However, above a certain temperature, active structure expands up to a length at which the temperature FBGs start to get elongated. Therefore, above this threshold, it is the thermal expansion of the plastic material that drives the response. Thereby, the response increases more rapidly with temperature. Since the response was linear in both regimes, it has been modeled by two intersecting planes, with different slopes, whose coefficients were estimated by LSE interpolation.

Finally, the sensitivity and cross-sensitivity of the two FBGs to pressure and temperature are given by the partial derivatives of the interpolating functions, $f_{P}(P, T)$ and $f_{T}(P, T)$, with respect to the pressure and temperature, respectively. Fig. 6a shows, for each sensor, the pressure sensitivity of FBGP, while Fig. 6b shows its temperature cross-sensitivity.

The sensors $S_{1}, S_{2}$ and $S_{3}$ showed a fair repeatability in terms of pressure response. In general, all three shared a similar behavior and an average pressure sensitivity for the pressure FBG from approx. 18 to $28 \mathrm{pm} / \mathrm{cm} \mathrm{H}_{2} \mathrm{O}$ with an absolute sensitivity variation of $5 \mathrm{pm} / \mathrm{cm}$ circa over the entire pressure range. The sensitivity decreases with the pressure as the transducer's wedges start to bend at high pressure, and the transducer becomes less effective in transferring the external pressure to the fiber. The differences among these sensors are due to the inhomogeneity accidentally introduced during the implementation of each prototype. In particular, the different strain with which the FBGp was anchored to the supporting walls, plays a key role. For the same reason, the sensor $E_{1}$ is characterized by a larger average sensitivity with a broader variation in the extended range of calibration, given the large pre-strain that was applied to the FBGp during implementation. For all the sensors, the average cross-sensitivity to temperature of $\mathrm{FBG}_{\mathrm{P}}$ ranges from about 150 to about $200 \mathrm{pm} /{ }^{\circ} \mathrm{C}$ with a sensitivity that varies over the calibration range of several $\mathrm{pm} /{ }^{\circ} \mathrm{C}$. Again, this is likely due to the different pre-strain applied to FBGp. In particular, the specific strain condition of FBGP, along with the temperature dependence of the mechanical properties of the plastic material of the transducer, determines also the slope of the sensitivities contour lines of $f_{P}(P, T)$ with respect to the temperature.

A similar graph for the temperature FBG is scarcely informative and not shown here, given that the fitting model is linear. For all QDPSs, the temperature sensitivity of the $\mathrm{FBG}_{\mathrm{T}}$ was about $13 \mathrm{pm} /{ }^{\circ} \mathrm{C}$, close to that of a bare $\mathrm{FBG}$, at lower temperatures (where the interpolating planes shown in Fig. $5 b$ are less steep). Instead, the different QDPSs had large sensitivity of the $\mathrm{FBG}_{\mathrm{T}}$ at higher temperatures, ranging from about 60 to about $130 \mathrm{pm} /{ }^{\circ} \mathrm{C}$, where the high thermal expansion of the transducer's polymer boosts the sensitivity. Instead, the variability of the sensitivity among the different sensors is likely due to the inhomogeneities of the anchoring condition of the fiber during the implementation. These sensitivities confirm the hypothesis, introduced above, that the response of the FBGs is driven by the thermo-optic effect at lower temperatures and by the thermal expansion of polymer at higher ones. The cross-sensitivity of the $\mathrm{FBG}_{\mathrm{T}}$ to pressure was below $0.5 \mathrm{pm} / \mathrm{cm} \mathrm{H}_{2} \mathrm{O}$ in all sensors. 

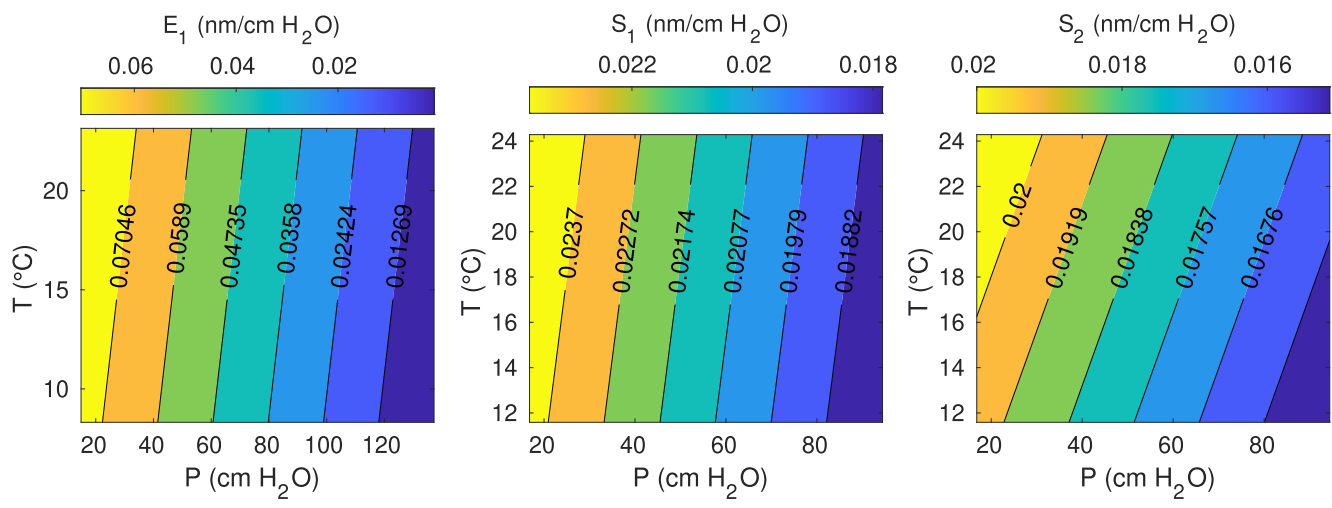

(a)
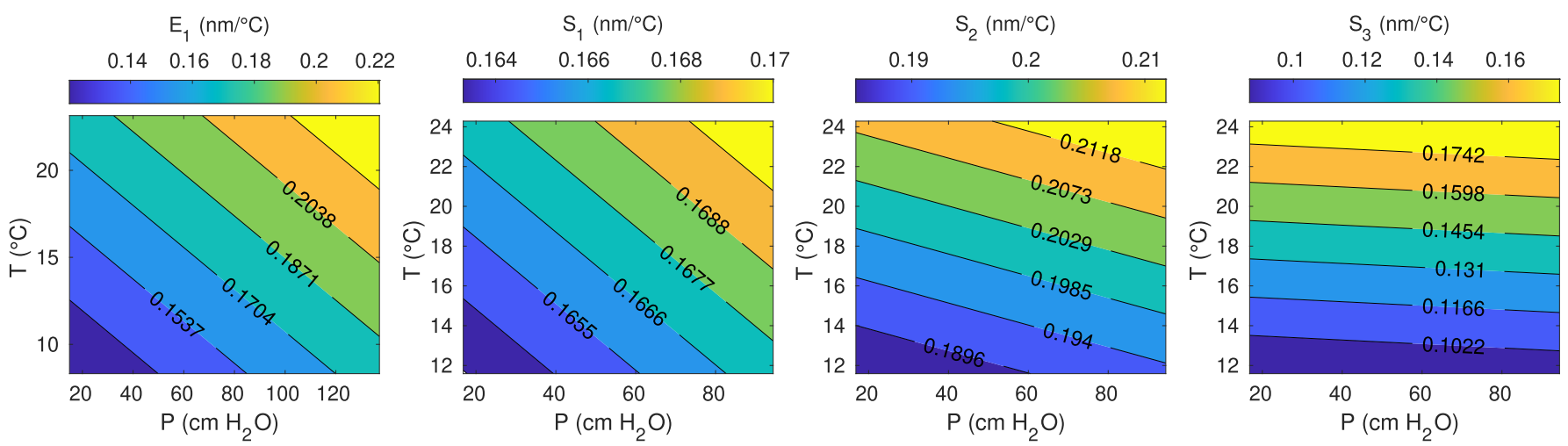

(b)

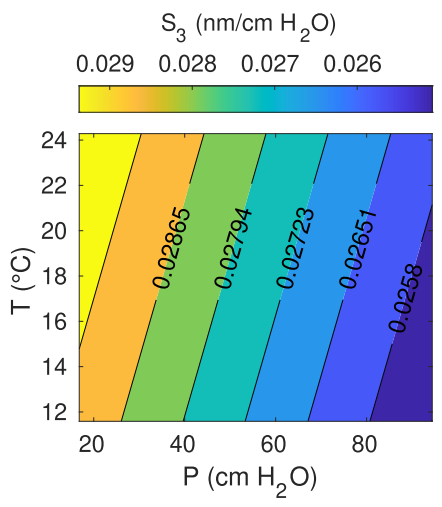

Fig. 6. Sensitivity to pressure (a) and temperature (b) of the pressure FBG, i.e., derivative of $f_{P}(P, T)$ with respect to pressure $P$ and temperature $T$, respectively, for all the sensors.

Overall, the accuracy of the sensors, estimated as the product of the sensitivities with the corresponding interpolation residuals, was $5 \mathrm{~cm}$ on the water level and $0.5^{\circ} \mathrm{C}$ on the temperature for all the sensors.

The last step of the analysis was the calculation of the calibration surfaces of the sensors, i.e., the inverse functions $P=g_{P}\left(\lambda_{P}, \lambda_{T}\right)$ and $T=g_{T}\left(\lambda_{P}, \lambda_{T}\right)$ that map the wavelength shift of the FBGs into pressure and temperature values. Due to the nonlinearity of $f_{P}(T, P)$, the calibration surfaces were calculated by inverting the 2-dimensional 2 -variate function $f(P, T)=\left[f_{P}(P, T), f_{T}(P, T)\right]$ numerically; the results are shown in Fig. 7a and 7b for the pressure and temperature, respectively.

As a general comment about the bandwidth allocation, given that several sensors of this type could be daisy-chained in an array, some attention should be paid to the choice of the FBGs' central wavelengths. As the pressure increases, the central wavelength of FBGp increases as well; this may limit the measurement range of the concatenated sensors. Considering the average bandwidth occupied by the implemented sensors, a minimum guard interval of $8 \mathrm{~nm}$ between the CWL of FBGP and the CWL of $\mathrm{FBG}_{\mathrm{T}}$ of another sensor in the same array should be kept to guarantee a pressure range of about 1.5-2 m of water head.

\section{FIELD TEST}

We arranged and performed a large scale experiment for dike monitoring with the QDPSs, which occurred in Delft,
The Netherlands, at the test facility of FloodProofHolland. This site consists of a series of basins, adjacent to each other, that can be filled or emptied at will. The four QDPSs sensors have been installed at the FloodProofHolland facility about one month before the experiment was run; this has been done to let the soil to compact and avoid creating unwanted preferential flow paths for water infiltration.

As schematically shown in Fig. 8, the QDPSs were buried at different depths in vertical bores along a section of the dike; Fig. 9a shows the actual basin with an approximate indication of the positions of the QDPS. Sensors $S_{2}$ and $S_{3}$ were installed in the same bore for comparison, side by side. Next to each QDPS, about $0.5 \mathrm{~m}$ apart and approximately at the same depth, an electronic water pressure and temperature sensor (DIVER ${ }^{\circledR}$ - Van Essen) were installed in open standpipes for reference (the gray tubes indicated by the arrows in Fig. 9a); two additional standpipes with electronic water pressure and temperature sensor were installed at the two slopes of the dike, and one extra barometric pressure sensors $\left(\right.$ BARO $^{\circledR}$ - Van Essen) was used to monitor air pressure and temperature.

Before installation, the QDPS were wrapped in geotextile, as shown in Fig. 9b, which acted as a filter for the fine soil particles (approx permeability $400 \mathrm{l} / \mathrm{m}^{2} / \mathrm{s}$ ); then, they were inserted in the vertical bores and covered with a mixture of dug soil and bentonite (i.e., water swelling clay). At the time of the installation, The Netherlands was experiencing a period of unusual drought; therefore, the soil was dry, which 

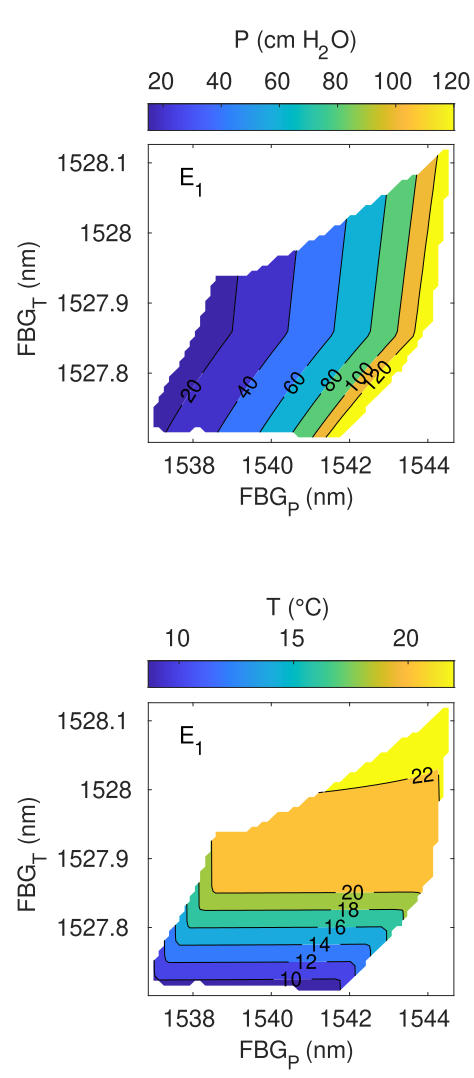

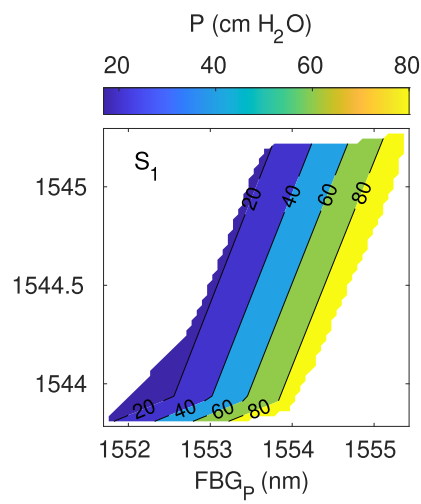

(a)

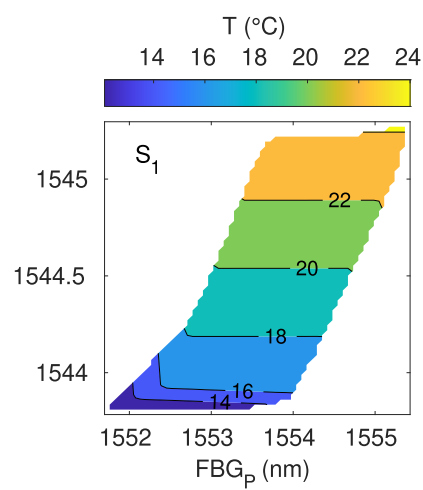

(a)
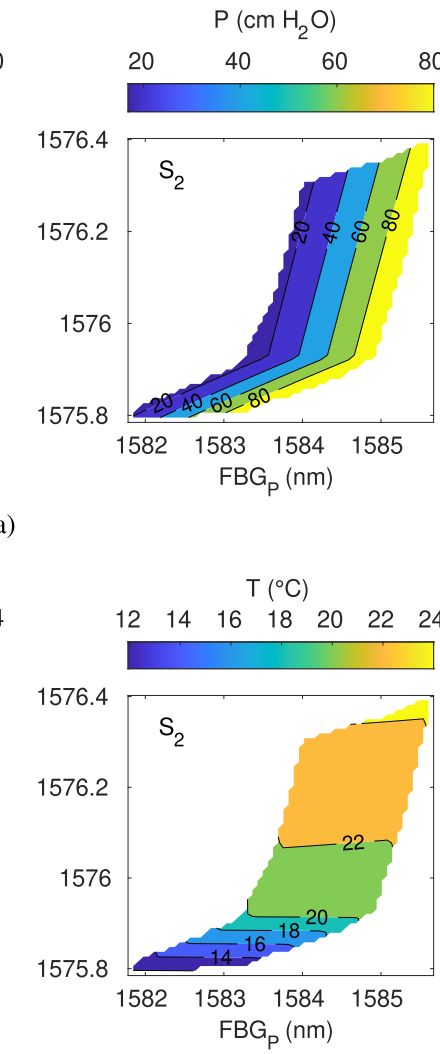

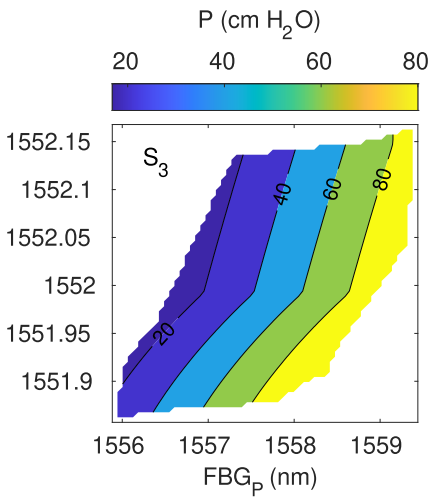

$\mathrm{T}\left({ }^{\circ} \mathrm{C}\right)$

$\begin{array}{lllllll}12 & 14 & 16 & 18 & 20 & 22 & 24\end{array}$

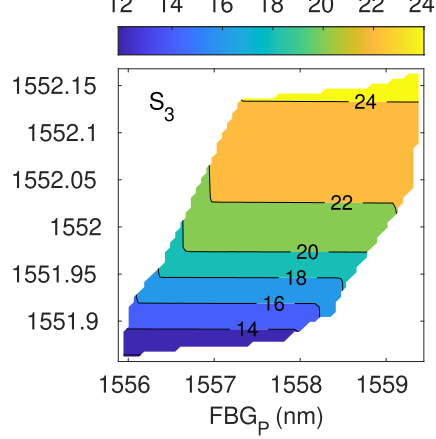

(b)

Fig. 7. Contour plots of the calibration surfaces for pressure (a) temperature (b), respectively.

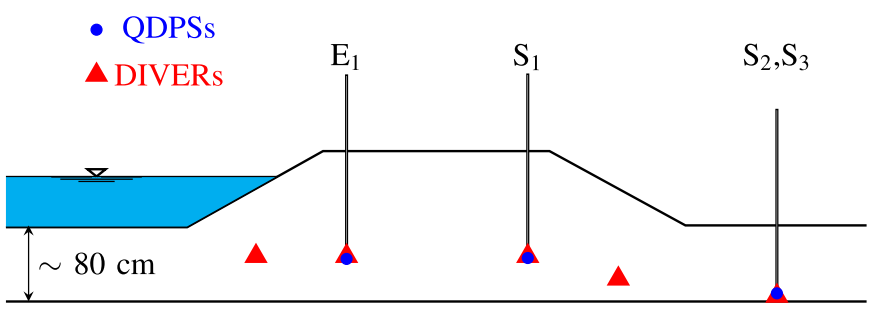

\begin{tabular}{c|ccc} 
Depth $(\mathrm{cm})$ & $\mathrm{E}_{1}$ & $\mathrm{~S}_{1}$ & $\mathrm{~S}_{2}, \mathrm{~S}_{3}$ \\
\hline w.r.t. wet side & -35 & -34 & -73 \\
w.r.t. soil top & -119 & -118 & -75
\end{tabular}

Fig. 8. Schematic of the QDPSs and DIVERs installation with respect to the dike (drawing not in scale). In the inset, the installation depths of each QDPSs.

made digging and filling the bores quite difficult. To easy the operation, the bores were watered and the soil was pushed back in the bore with the aid of sticks.

The experiment consisted of filling and emptying the basin in a controlled manner over different cycles while monitoring water infiltration with the QDPSs and DIVERs. Specifically, the experiment lasted for more than 50 hours, and we implemented three filling and emptying cycles to assess the reliability and repeatability of the sensors over subsequent pressure ramps. During this field experiment, an additional interrogator, available on site, was either employed (National Instrument ${ }^{\circledR}$ PXIe-4844). The pressure variations successfully recorded by the four QDPS are shown in Fig. 10. There

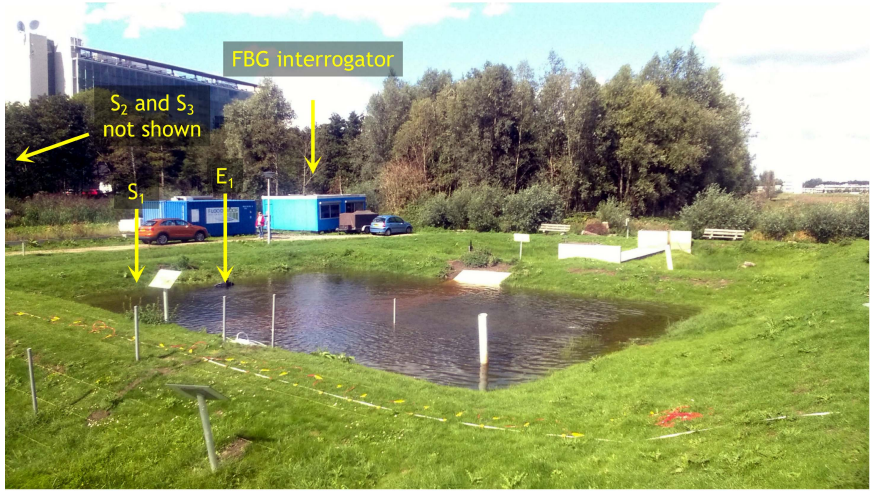

(a)

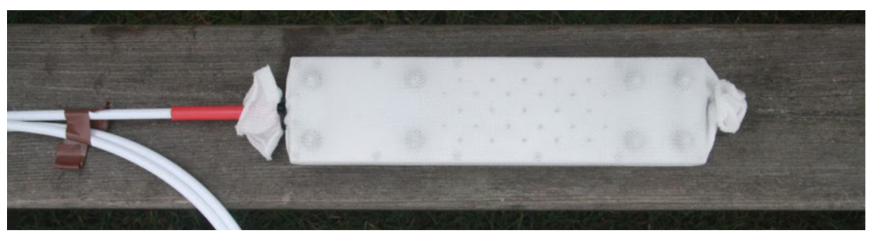

(b)

Fig. 9. (a) Picture of the experiment site with the basin fill of water; the interrogators were installed in the blue container visible in the background. (b) A sensor, wrapped by the geotextile.

was a reasonable agreement between the QDPSs and the corresponding DIVERs for all the three filling and emptying ramps with similar trends, spikes, and timing. The relative pressure steps measured by the QDPS and the corresponding electrical sensor match well, within few centimeters, while the 


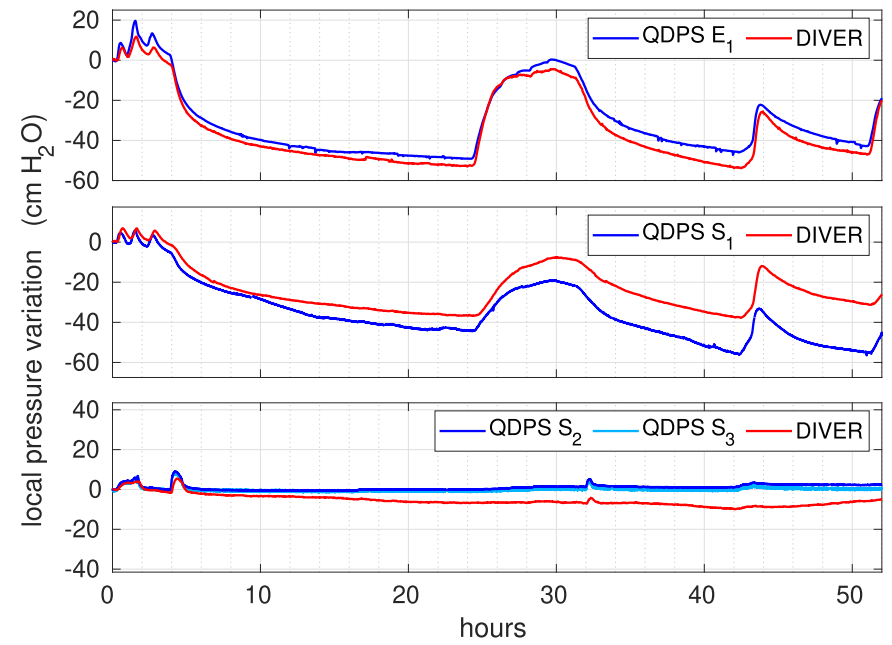

Fig. 10. Pressure variation recorded by the QDPSs, and by the corresponding DIVERs. The comparison can be only qualitative because the DIVERs were installed differently and at a slightly different location. Graphs have the same time scale.

absolute water head measured by the sensor $E_{1}$ is approx. $5 \mathrm{~cm}$ higher than that of the corresponding DIVER, on average, over almost the entire experiment. On the contrary, the gap between the water head measured by sensor $S_{1}$ and the DIVER tends to increase over time, up to $20 \mathrm{~cm}$, with the DIVER showing larger values.

At the far-field side, the sensors $S_{2}$ and $S_{3}$ show an almost constant water level, with some spikes at the end of the filling of the basin or shortly after. This behavior is consistent with the fact that being quite far from the levee body, they were expected to have a slower response as the soil conductivity represents a non-linear head loss behavior of the groundwater system, making locations that are further to present significantly lower pressure head variations. On the contrary, the water level measured by the corresponding DIVER, while sharing the same peaks, shows a decreasing trend.

The quantitive differences in the response of QDPSs and DIVERs may be explained by the different installation (with the QDPSs buried in the soil and the DIVERs installed in open standpipes) and by a small displacement of about $50 \mathrm{~cm}$ in their positioning with respect to the DIVERs. In particular, the relative different installation positions might have had the larger impact because the dike had been built with highly inhomogeneous soil and material to mimic the oldest Dutch dikes' structure. Nonetheless, as a proof of physical reproducibility, there is also an excellent agreement between the pressure variations recorded by QDPS $S_{2}$ and $S_{3}$ (which were installed at the same location), although sensor $\mathrm{S}_{3}$ provided noisier data (cyan-coloured curve of the lower plot in Fig. 10) because it was damage by the mechanical action of the sticks for pushing the soil into the bore during installation.

\section{CONCLUSION}

This paper presented a rugged temperature compensated FBG-based sensor for pressure measurements in soil, employing a 3D-printed transducer and a metallic case capable of withstanding harsh environmental conditions. The sensor encases two FBGs: the first FBG, embedded in an engineered pantograph, is devoted to the pressure measurements. Thanks to the transducing efficiency, it has a high sensitivity of several $\mathrm{pm} / \mathrm{cm} \mathrm{H}_{2} \mathrm{O}$. Due to the high thermal expansion of the transducer material, it shows a relevant temperature sensitivity. This cross-sensitivity is effectively compensated by a second FBG, hosted in a separated adjacent structure, almost pressure insensitive. Overall, the sensor accuracy is $5 \mathrm{~cm}$ and $0.5{ }^{\circ} \mathrm{C}$ for pressure and temperature, respectively.

We validated the design in a real scenario by installing four sensors, for over a month, in a real scale dike at the Water Proof Holland facility in Deft, Netherlands. During the experiment, which lasted more than two days, the artificial basin was filled and emptied three times. The pressure at different positions in the levee cross-section was measured by the QDPS sensors and by electric sensors installed sideby-side as a reference. The experimental results provided by the optical fiber sensors are nicely reproducible and consistent with those from electronic reference sensors.

\section{ACKNOWLEDGMENT}

This is an expanded article from the 7th European Workshop on Optical Fibre Sensors.

\section{RefERENCES}

[1] C. Cavalloni and J. von Berg, "Overview: Principles and technologies for pressure sensors for automotive applications," in Advanced Microsystems for Automotive Applications Yearbook, S. Krueger and W. Gessner, Eds. Berlin, Germany: Springer, 2002, pp. 232-242.

[2] S. Sikarwar, Satyendra, S. Singh, and B. C. Yadav, "Review on pressure sensors for structural health monitoring," Photonic Sensors, vol. 7, no. 4, pp. 294-304, Dec. 2017.

[3] F. Xu et al., "Recent developments for flexible pressure sensors: A review," Micromachines, vol. 9, no. 11, p. 580, 2018.

[4] A. G. Leal-Junior et al., "Wearable and fully-portable smart garment for mechanical perturbation detection with nanoparticles optical fibers," IEEE Sensors J., vol. 21, no. 3, pp. 2995-3003, Feb. 2021.

[5] J. M. Hooke, "An analysis of the processes of river bank erosion," J. Hydrol., vol. 42, nos. 1-2, pp. 39-62, 1979.

[6] E. W. Lane, "Security from under-seepage-masonry dams on Earth foundations," Trans. Amer. Soc. Civil Eng., vol. 100, no. 1, pp. 1235-1272, Jan. 1935.

[7] W. J. Klerk, T. Schweckendiek, F. den Heijer, and M. Kok, "Value of information of structural health monitoring in asset management of flood defences," Infrastructures, vol. 4, no. 3, p. 56, Aug. 2019.

[8] M. Wewer, J. P. Aguilar-López, M. Kok, and T. Bogaard, "A transient backward erosion piping model based on laminar flow transport equations," Comput. Geotechnics, vol. 132, Apr. 2021, Art. no. 103992. [Online]. Available: https://www.sciencedirect.com/science/article/ pii/S0266352X20305553

[9] L. Schenato, "A review of distributed fibre optic sensors for geohydrological applications," Appl. Sci., vol. 7, no. 9, p. 896, Sep. 2017.

[10] M. Shanafield, E. W. Banks, J. W. Arkwright, and M. B. Hausner, "Fiber-optic sensing for environmental applications: Where we have come from and what is possible," Water Resour. Res., vol. 54, no. 11, pp. 8552-8557, Nov. 2018.

[11] M. A. Riza, Y. I. Go, S. W. Harun, and R. R. J. Maier, "FBG sensors for environmental and biochemical applications-A review," IEEE Sensors J., vol. 20, no. 14, pp. 7614-7627, Jul. 2020.

[12] Q. Yu and X. Zhou, "Pressure sensor based on the fiber-optic extrinsic Fabry-Pérot interferometer," Photonic Sensors, vol. 1, no. 1, pp. 72-83, Nov. 2010.

[13] X. Zhou, Q. Yu, and W. Peng, "Fiber-optic Fabry-Pérot pressure sensor for down-hole application," Opt. Lasers Eng., vol. 121, pp. 289-299, Oct. 2019.

[14] E. Vorathin, Z. M. Hafizi, N. Ismail, and M. Loman, "Review of high sensitivity fibre-optic pressure sensors for low pressure sensing," Opt. Laser Technol., vol. 121, Jan. 2020, Art. no. 105841. 
[15] S. Mikhailov, L. Zhang, T. Geernaert, F. Berghmans, and L. Thevenaz, "Distributed hydrostatic pressure measurement using phase-OTDR in a highly birefringent photonic crystal fiber,' J. Lightw. Technol., vol. 37, no. 18, pp. 4496-4500, Sep. 15, 2019.

[16] L. Schenato, A. Galtarossa, A. Pasuto, and L. Palmieri, "Distributed optical fiber pressure sensors," Opt. Fiber Technol., vol. 58, Sep. 2020, Art. no. 102239

[17] L. Schenato, A. Pasuto, A. Galtarossa, and L. Palmieri, "An optical fiber distributed pressure sensing cable with Pa-sensitivity and enhanced spatial resolution," IEEE Sensors J., vol. 20, no. 11, pp. 5900-5908, Jun. 2020.

[18] S. J. Mihailov, "Fiber Bragg grating sensors for harsh environments," Sensors, vol. 12, no. 2, pp. 1898-1918, Feb. 2012

[19] L. Reekie, Y. T. Chow, and J. P. Dakin, "Optical in-fibre grating high pressure sensor," Electron. Lett., vol. 29, no. 4, pp. 398-399, 1993.

[20] L. Liu, H. Zhang, Q. Zhao, Y. Liu, and F. Li, "Temperature-independent FBG pressure sensor with high sensitivity," Opt. Fiber Technol., vol. 13, no. 1, pp. 78-80, Jan. 2007.

[21] Q. Wen, J. Zhu, S. Gong, J. Huang, H. Gu, and P. Zhao, "Design and synthesis of a packaging polymer enhancing the sensitivity of fiber grating pressure sensor," Prog. Natural Sci., vol. 18, no. 2, pp. 197-200, Feb. 2008.

[22] D. Sengupta, M. S. Shankar, P. S. Reddy, R. L. N. S. Prasad, and K. Srimannarayana, "Sensing of hydrostatic pressure using FBG sensor for liquid level measurement," Microw. Opt. Technol. Lett., vol. 54, no. 7, pp. 1679-1683, Jul. 2012.

[23] L. Schenato, R. Aneesh, L. Palmieri, A. Galtarossa, and A. Pasuto, "Fiber optic sensor for hydrostatic pressure and temperature measurement in riverbanks monitoring," Opt. Laser Technol., vol. 82, pp. 57-62, Aug. 2016.

[24] M. Liu, Y. Wu, C. Du, D. Jiang, and Z. Wang, "FBG-based liquid pressure sensor for distributed measurement with a single channel in liquid environment," IEEE Sensors J., vol. 20, no. 16, pp. 9155-9161, Aug. 2020.

[25] D. Song, "Liquid-level sensor using a fiber Bragg grating and carbon fiber composite diaphragm," Opt. Eng., vol. 50, no. 1, Jan. 2011, Art. no. 014401.

[26] J. Huang, Z. Zhou, X. Wen, and D. Zhang, "A diaphragm-type fiber Bragg grating pressure sensor with temperature compensation," Measurement, vol. 46, no. 3, pp. 1041-1046, Apr. 2013.

[27] Y.-F. Gu, Y. Zhao, R.-Q. Lv, and Y. Yang, "A practical FBG sensor based on a thin-walled cylinder for hydraulic pressure measurement," IEEE Photon. Technol. Lett., vol. 28, no. 22, pp. 2569-2572. Nov. 15, 2016.

[28] Z. Hong-kun, Z. Yong, Z. Qiang, and L. Ri-qing, "High sensitivity optical fiber pressure sensor based on thin-walled oval cylinder," Sens. Actuators A, Phys., vol. 310, Aug. 2020, Art. no. 112042.

[29] R. Ishikawa et al., "Pressure dependence of fiber Bragg grating inscribed in perfluorinated polymer fiber," IEEE Photon. Technol. Lett., vol. 29, no. 24 , pp. 2167-2170, Dec. $15,2017$.
[30] A. Leal-Junior et al., "Characterization of a new polymer optical fiber with enhanced sensing capabilities using a Bragg grating," Opt. Lett., vol. 43, no. 19, pp. 4799-4802, Oct. 2018.

[31] A. Leal-Junior, V. Campos, A. Frizera, and C. Marques, "Low-cost and high-resolution pressure sensors using highly stretchable polymer optical fibers," Mater. Lett., vol. 271, Jul. 2020, Art. no. 127810. [Online]. Available: https://www.sciencedirect.com/science/article/pii/ S0167577X20305152

[32] W. Zhang, F. Li, and Y. Liu, "FBG pressure sensor based on the double shell cylinder with temperature compensation," Measurement, vol. 42, no. 3, pp. 408-411, Apr. 2009.

[33] F. Urban, J. Kadlec, R. Vlach, and R. Kuchta, "Design of a pressure sensor based on optical fiber Bragg grating lateral deformation," Sensors, vol. 10, no. 12, pp. 11212-11225, Dec. 2010.

[34] A. Leal-Junior, A. Frizera, C. Díaz, C. Marques, M. Ribeiro, and M. J. Pontes, "Material features based compensation technique for the temperature effects in a polymer diaphragm-based FBG pressure sensor,' Opt. Exp., vol. 26, no. 16, pp. 20590-20602, 2018.

[35] A. G. Leal-Junior, C. A. R. Díaz, A. Frizera, C. Marques, M. R. N. Ribeiro, and M. J. Pontes, "Simultaneous measurement of pressure and temperature with a single FBG embedded in a polymer diaphragm," Opt. Laser Technol., vol. 112, pp. 77-84, Apr. 2019. [Online]. Available: https://www.sciencedirect.com/science/article/pii/ S0030399218312003

[36] Y. Liu, Z. Guo, Y. Zhang, K. S. Chiang, and X. Dong, "Simultaneous pressure and temperature measurement with polymer-coated fibre Bragg grating," Electron. Lett., vol. 36, no. 6, p. 564, 2000.

[37] H.-J. Sheng, W.-F. Liu, K.-R. Lin, S.-S. Bor, and M.-Y. Fu, "Highsensitivity temperature-independent differential pressure sensor using fiber Bragg gratings," Opt. Exp., vol. 16, no. 20, 2008, Art. no. 16013.

[38] A. Leal-Junior, A. Frizera, and C. Marques, "A fiber Bragg gratings pair embedded in a polyurethane diaphragm: Towards a temperatureinsensitive pressure sensor," Opt. Laser Technol., vol. 131, Nov. 2020, Art. no. 106440.

[39] Y. K. Lin, T. S. Hsieh, L. Tsai, S. H. Wang, and C. C. Chiang, "Using three-dimensional printing technology to produce a novel optical fiber Bragg grating pressure sensor," Sensors Mater, vol. 28, no. 5, pp. 389-394, 2016.

[40] C. Hong, Y. Zhang, and L. Borana, "Design, fabrication and testing of a 3D printed FBG pressure sensor," IEEE Access, vol. 7, pp. 38577-38583, 2019.

[41] C. Hong, Y. Yuan, Y. Yang, Y. Zhang, and Z. A. Abro, "A simple FBG pressure sensor fabricated using fused deposition modelling process," Sens. Actuators A, Phys., vol. 285, pp. 269-274, Jan. 2019.

[42] L. Schenato, J. P. A. López, A. Galtarossa, A. Pasuto, T. Bogaard, and L. Palmieri, "Design and field testing of a fiber optic pressure sensor for underground water level monitoring," Proc. SPIE, vol. 11199, pp. 93-96, Aug. 2019.

[43] L. Schenato et al., "Highly sensitive FBG pressure sensor based on a 3Dprinted transducer," J. Lightw. Technol., vol. 37, no. 18, pp. 4784-4790, Sep. 15, 2019. 